\title{
Preventive Long-Term Effects of a Topical Film-Forming Medical Device with Ultra-High UV Protection Filters and DNA Repair Enzyme in Xeroderma Pigmentosum: A Retrospective Study of Eight Cases
}

\author{
Sandra Giustini ${ }^{a} \quad E^{2}$ manuele Miraglia ${ }^{a} \quad$ Enzo Berardesca ${ }^{b}$ \\ Massimo Milani $^{\mathrm{c}}$ Stefano Calvieri ${ }^{\mathrm{a}}$ \\ ${ }^{a}$ Genodermatosis Service, Dermatology Clinic, University 'La Sapienza', Rome, \\ ${ }^{\mathrm{b}} \mathrm{San}$ Gallicano Dermatological Institute, Rome, and 'Medical Direction Isdin Italy, \\ Milan, Italy
}

\section{Key Words}

Xeroderma pigmentosum $\cdot$ Photolyase $\cdot$ Non-melanoma skin cancer $\cdot$ Sunscreen

\begin{abstract}
Skin cancer is common in xeroderma pigmentosum (XP) due to a DNA repair mechanisms genetic defect. Ultraviolet (UV) exposure is the main cause of increased incidence of actinic keratosis (AK), basal cell carcinoma (BCC) and squamous cell carcinoma (SCC) observed in XP subjects. Photoprotection is therefore a mandatory strategy in order to reduce skin damage. A topical DNA repair enzyme has been shown to slow down the development of skin lesions in XP. However, there are no data regarding the effects of photoprotection combined with DNA repair strategies in this clinical setting. A film-forming medical device containing the DNA repair enzyme photolyase and very high-protection UV filters (Eryfotona AK-NMSC, Ery) is currently available. We report retrospective data regarding the use of Ery in 8 patients (5 women, 3 men) with a diagnosis of XP treated for at least 12 consecutive months, comparing the rate of new skin lesions (AK, BCC and SCC) during active treatment with Ery and during 12 months just before the use of the product. New AK, BCC and SCC mean lesion numbers during the 1-year Ery treatment were 5, 3 and 0, respectively in comparison with 14, 6.8 and 3 lesions, respectively during the 1-year pre-treatment period. Ery use was associated with a $65 \%$ reduction in appearance of new AK lesions and with 56 and 100\% reductions in the
\end{abstract}


Giustini et al.: Preventive Long-Term Effects of a Topical Film-Forming Medical Device with Ultra-High UV Protection Filters and DNA Repair Enzyme in Xeroderma

Pigmentosum: A Retrospective Study of Eight Cases

incidence of new BCC and SCC lesions, respectively. These data suggest that topical use of photoprotection and DNA repair enzyme could help lower skin cancer lesions in XP. Control prospective trials are advisable in this clinical setting.

(c) 2014 S. Karger AG, Basel

\section{Introduction}

Skin cancer is the most common form of cancer in white people [1]. Ultraviolet (UV) exposure is considered the principal cause of the pathogenesis of cancer skin lesions as a consequence of direct and indirect DNA damage [2]. The most common UV-induced DNA damage is the formation of cyclobutane pyrimidine dimers (CPDs). CPDs are in fact the major DNA photoproducts produced upon exposure to UV radiation. The carcinogenic effect of UV radiation, especially UVB but also UVA, relies on the production of these genotoxic photoproducts such as CPDs but also 6-4 photoproducts (6-4PPs) [3]. These UV-induced DNA alterations interfere with critical biological cell processes that are needed for cell viability, favoring skin cancer development.

Chronic UV exposure is the main cause of the increased incidence of actinic keratosis (AK), basal cell carcinoma (BCC) and squamous cell carcinoma (SCC) observed in subjects suffering from xeroderma pigmentosum (XP) [4]. XP is a rare genetic disease with clinical and cellular hypersensitivity to UV radiation, characterized by a defective DNA repair process [5]. Patients with this disease experience sun-induced cutaneous and eye abnormalities [6]. In XP patients sun exposure causes cumulative DNA damage where the formation of $\mathrm{CPD}$ and 6-4PP is not efficiently repaired [7]. Photoprotection is therefore a mandatory and vital preventive strategy in order to reduce skin damage in subjects with XP. Patients must avoid all intentional exposure to the sun and to artificial UV radiation and must also protect themselves against indirect exposure to UV light. From a theoretical point of view, strategies aimed to improve the efficiency of UV-induced DNA damage repair could be considered an important therapeutic goal. In 2001, Yarosh et al. [8] showed that topical DNA repair enzyme (endonuclease) application is able to lower the development of skin lesions in XP. Twelvemonth administration of this product decreased the appearance rate of new AK, BCC and SCC lesions. However, there are no data regarding the effect of a combined photoprotection and DNA repair strategy in this clinical setting. Eryfotona AK-NMSC (Ery) (Isdin Sa, Barcelona, Spain) is a film-forming medical device containing the DNA repair enzyme photolyase and very high-protection (100+) UV filters (repairsomes). Photolyases are monomeric enzymes, not present in placental mammals, which specifically recognize and repair either CPDs or 64PPs [9]. CPD-repairing photolyase is a light-activated flavoenzyme that binds to pyrimidine dimers in DNA and repairs them in a reaction triggered by electron transfer from the photoexcited flavin cofactor to the dimer [10]. Experimental studies carried out in patients with AK have shown that application of this medical device is associated with an improvement of the cancerization field evaluated both clinically [11] and with confocal microscopy [12]. Due to its high photoprotection level (SPF 100+ and high UVA protection) and photorepair potential, this medical device could be considered a suitable supportive strategy in addition to general sun protection measures in patients with XP.

\section{Study Design and Methods}

We performed a retrospective case study in 8 patients, 5 women and 3 men, mean age 55 years, who had a diagnosis of XP confirmed by DNA synthesis assay. All patients had been 
Giustini et al.: Preventive Long-Term Effects of a Topical Film-Forming Medical Device with Ultra-High UV Protection Filters and DNA Repair Enzyme in Xeroderma

Pigmentosum: A Retrospective Study of Eight Cases

visiting our centers since the diagnosis of XP was made. For the inclusion in this case series subjects had to fulfil the following criteria: availability of detailed and complete clinical and surgical data records with a documented history of the disease (number of lesions, number and type of interventions for skin lesions), a positive history of 5 or more skin lesions in the past (AK, BCC or SCC) and at least one consecutive year of treatment with Ery, applied twice daily. During the Ery application patients were not to have been using other forms of topical sun protection products. Particular attention was paid to checking that all other protective measures were comparable between the two periods evaluated. For skin lesions detailed histological records had to be available. The study was performed in two tertiary-level genodermatosis clinics (University La Sapienza, Rome and San Gallicano Institute, Rome) with specific experience and training in XP management. All subjects gave their informed consent to the collection of the data for the study.

\section{Results}

In this case series retrospective analysis we collected data of 8 subjects ( 5 men, 3 women; mean age 55 years). Patient characteristics are summarized in table 1 . All patients had a genetically confirmed XP diagnosis. In the year before Ery application the mean number of lesions registered was 14 AK lesions, 6.8 BCC lesions and 3 SCC lesions. All lesions were classified according to histological and/or clinical records. During the 1-year Ery treatment the mean rate of new AK, BCC and SCC lesions was 5, 3 and 0, respectively (fig. 1). Ery use was therefore associated with a $65 \%$ reduction in the appearance of new AK lesions and with 56 and $100 \%$ reductions in the incidence of new BCC and SCC lesions, respectively. The product was in general well tolerated in these patients. No relevant side effects were reported.

\section{Discussion and Conclusion}

XP patients have a genetic defect in the DNA repair system [5]. This alteration causes the lack of an efficient repair process of UV-induced DNA damage. As a result of this alteration, the rates of pre-malignant and malignant skin lesions, such as AK, BCC and SCC, are roughly 1,000 times higher than in general population [13]. In general the treatment of BCC and SCC consists in Mohs micrographic surgery. Regarding AK lesions both lesion-targeted (cryosurgery, curettage) and field-targeted treatments (photodynamic therapy, imiquimod, ingenol, etc.) are commonly used [14]. As preventive strategies the use of sunscreens has been shown to reduce the risk of new AK. Yarosh et al. [8] in 2001 showed for the first time that the use of endonuclease, a DNA repair enzyme, in a liposome formulation was able to reduce the appearance of UV-induced skin lesions in XP patients. Photolyase is another very effective DNA repair enzyme. It is able to rapidly repair specifically UV-induced DNA damage [9]. Photolyase specifically converts dimerized pyrimidines back into their original monomeric form. In order to induce DNA repair in the epidermis of skin treated with photolyase, exposure to 300-500 nm light is needed in order to photoreactivate the enzyme. A clinical study conducted by Stege et al. [15] showed that after exposure to UVB radiation, the application of topical photolyase prevented radiation-associated immunosuppression and erythema formation. Clinical studies carried out in patients with AK have shown that application of photolyase combined with sunscreen filters is associated with an improvement of the cancerization field evaluated both clinically and with confocal microscopy [11, 
Giustini et al.: Preventive Long-Term Effects of a Topical Film-Forming Medical Device with Ultra-High UV Protection Filters and DNA Repair Enzyme in Xeroderma Pigmentosum: A Retrospective Study of Eight Cases

12]. In AK patients treated with photodynamic therapy, the application of this product for 9 months was associated with a significantly lower number of new AK lesions in comparison with a sunscreen not containing photolyase [16].

Photoprotection and photorepair could be a useful strategy in order to further reduce the risk of pre-malignant and malignant UV-induced skin lesions in comparison with photoprotection only strategies. This cases series retrospective study suggests that the use of a photoprotective and photorepair topical product is associated with a relevant reduction in the rate of new appearing skin lesions in XP patients (65\% reduction in new AK lesions; 56 and $100 \%$ reductions in new BCC and SCC lesions, respectively). These clinical results look quite similar to the data obtained by Yarosh et al. [8], who in a 12-month period observed a 69\% reduction in new AK lesions and a 30\% reduction in BCC lesions in XP subjects treated with the DNA repair enzyme endonuclease. In their study no differences were observed regarding new SCC lesions between the active and the placebo group. Our retrospective data could be useful in order to plan future prospective controlled trials with the aim to evaluate the protective and preventive role of this topical product.

\section{Disclosure Statement}

Massimo Milani is an Isdin employee. He collaborated in the final version of the manuscript, but was not involved in data collection and clinical data analysis.

\section{References}

1 Kraemer K: Sunlight and skin cancer. Proc Natl Acad Sci USA 1997;94:11-14.

-2 Griffiths HR, Mistry P, Herbert KE, Lunec J: Molecular and cellular effects of ultraviolet light-induced genotoxicity. Crit Rev Clin Lab Sci 1998;35:189-237.

-3 Vink AA, Roza L: Biological consequences of cyclobutane pyrimidine dimers. J Photochem Photobiol 2001;65:101-104.

4 Kramer K, Lee M, Scotto J: Xeroderma pigmentosum. Arch Dermatol 1987;123:241-250.

5 Cleaver J: Defective repair replication of DNA in xeroderma pigmentosum. Nature 1968;218:652-656.

-6 Robbins JH, Kraemer KH, Lutzner MA: Xeroderma pigmentosum: an inherited disease with sun sensitivity, multiple cutaneous neoplasms, and abnormal DNA repair. Ann Intern Med 1974;80:221-248.

-7 Kleijer WJ, Laugel V, Berneburg M, Nardo T, Fawcett H, Gratchev A, Jaspers NG, Sarasin A, Stefanini M, Lehmann AR: Incidence of DNA repair deficiency disorders in western Europe: xeroderma pigmentosum, Cockayne syndrome and trichothiodystrophy. DNA Repair (Amst) 2008;7:744-750.

-8 Yarosh D, Klein J, O'Connor A, Hawk J, Rafal E, Wolf P: Effect of topically applied T4 endonuclease V in liposomes on skin cancer in xeroderma pigmentosum: a randomised study. Xeroderma Pigmentosum Study Group. Lancet 2001;357:926-929.

-9 Gerkema MP, Davies WI, Foster RG, Menaker M, Hut RA: The nocturnal bottleneck and the evolution of activity patterns in mammals. Proc Biol Sci 2013;280:20130508.

10 Schul W, Jans J, Rijksen YM, Klemann KH, Eker AP, de Wit J, Nikaido O, Nakajima S, Yasui A, Hoeijmakers JH, van der Horst GT: Enhanced repair of cyclobutane pyrimidine dimers and improved UV resistance in photolyase transgenic mice. EMBO J 2002;21:4719-4729.

11 Puviani M, Barcella A, Milani M: Efficacy of a photolyase-based device in the treatment of cancerization field in patients with actinic keratosis and non-melanoma skin cancer. G Ital Dermatol Venereol 2013;148:693698.

12 Puig-Butillé JA, Malvehy J, Potrony M: Role of CPI-17 in restoring skin homoeostasis in cutaneous field of cancerization: effects of topical application of a film-forming medical device containing photolyase and UV filters. Exp Dermatol 2013;22:494-496.

13 Lehmann AR, McGibbon D, Stefanini M: Xeroderma pigmentosum. Orphanet J Rare Dis 2011;6:70.

14 Dréno B, Amici JM, Basset-Seguin N, Cribier B, Claudel JP, Richard MA: Management of actinic keratosis: a practical report and treatment algorithm from AKTeam(TM) expert clinicians. J Eur Acad Dermatol Venereol 2014;28:1141-1149.

15 Stege H, Roza L, Vink AA, Grewe M, Ruzicka T, Grether-Beck S, Krutmann J: Enzyme plus light therapy to repair DNA damage in ultraviolet-B-irradiated human skin. Proc Natl Acad Sci USA 2000;97:1790-1795. 
Giustini et al.: Preventive Long-Term Effects of a Topical Film-Forming Medical Device with Ultra-High UV Protection Filters and DNA Repair Enzyme in Xeroderma Pigmentosum: A Retrospective Study of Eight Cases

16 Piaserico S, Milani M: Efficacia clinica della fotoliasi topica dopo terapia fotodinamica in soggetti con cheratosi attiniche: studio prospettico randomizzato intrapaziente. G Ital Dermatol Venereol 2012;147(suppl 2):109.

Table 1. Patient characteristics

\begin{tabular}{ll}
\hline Demography & \\
Age, mean \pm SD & $55 \pm 20$ \\
Women/men & $5 / 3$ \\
Age at XP diagnosis, mean \pm SD & $15 \pm 12$ \\
Skin type II/III & $6 / 2$ \\
Mean duration of Ery treatment, years & 3 \\
\hline
\end{tabular}

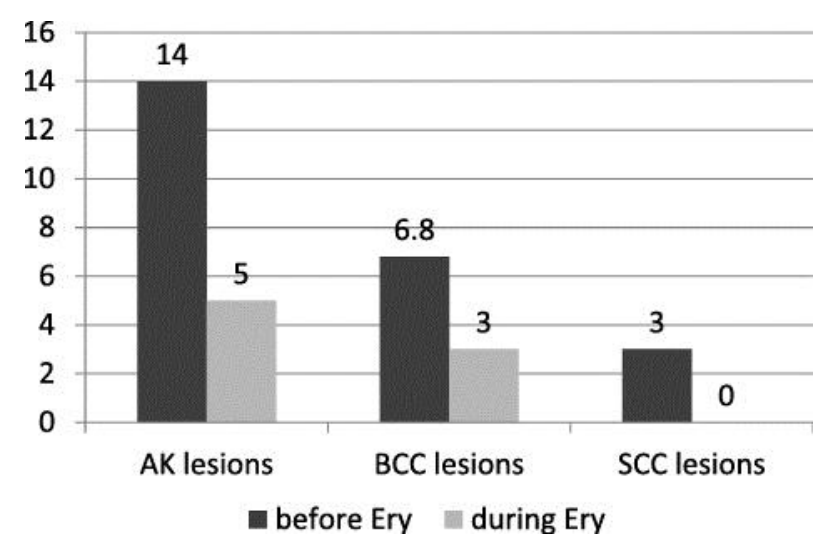

Fig. 1. Mean rate of skin lesions before and after Ery treatment (12-month time span). 